\title{
Post-thyroidectomy neck ultrasonography in patients with thyroid cancer and a review of the literature
}

\author{
Sumbul Zaheer ${ }^{1}$, MRCP, Andrew $\underline{T a n}^{1}$, FRCR, Ee Sin $\underline{A n g}^{1}$, FRCR, Kelvin SH Loke ${ }^{1}$, MRCP, Yung Hsiang Kao ${ }^{1}$, MRCP, \\ Anthony Goh $^{1}$, MBBS, MSc, Wai Yin Wong ${ }^{1}$, FRCR
}

\begin{abstract}
The importance of routine neck ultrasonography for the detection of unsuspected local or nodal recurrence of thyroid cancer following thyroidectomy (with or without neck dissection) is well documented in many journal articles and international guidelines. Herein, we present a pictorial summary of the sonographic features of benign and malignant central neck compartment nodules and cervical lymph nodes via a series of high-quality ultrasonographic images, with a review of the literature.
\end{abstract}

Keywords: neck ultrasonography, post-thyroidectomy, recurrence, surveillance, thyroid cancer

\section{INTRODUCTION}

The usefulness of routine neck ultrasonography (US) in detecting unsuspected local or nodal recurrence of thyroid cancer after thyroidectomy is well documented in journal articles $^{(1,2)}$ and international guidelines. ${ }^{(3,4)}$ This evaluation is conducted as part of the routine follow-up in patients post thyroidectomy. In view of the importance of neck US in this group of patients, it is crucial for practitioners to possess knowledge of both the normal and abnormal features of the post-thyroidectomy neck on US. In this pictorial essay, we present the sonographic features of the post-thyroidectomy neck, with examples derived from cases seen at our department, the Department of Nuclear Medicine and PET, Singapore General Hospital, Singapore.

In our department, patients with thyroid cancer who are deemed to be at a higher risk of recurrence following thyroidectomy (with or without neck dissection) are referred for treatment and follow-up. These patients usually undergo radioactive iodine (RAI) remnant ablation and continue to have long-term follow-up. In line with current guidelines, the majority of such patients would have at least a yearly neck US, from as early as six months after surgery, after the postoperative changes have stabilised. Herein, we present a pictorial summary of the sonographic features of benign and malignant central neck compartment nodules and cervical lymph nodes, as well as a review of the literature.

\section{GENERAL CONSIDERATIONS}

\section{Local extent of the problem}

Thyroid cancer is the 10th most common cancer among Singaporean women, according to the Singapore Cancer Registry Interim Annual Registry Report on the trends in cancer incidence in Singapore from 2005 to 2009. ${ }^{(5)}$ The age- standardised rate of thyroid cancer in Singapore is reported to be 8.5 per 100,000 annually. ${ }^{(6)}$ Thyroid cancer is operable in more than $90 \%$ of cases, and thyroidectomy remains the main effective modality of therapy. ${ }^{(3)}$

Papillary thyroid cancer (PTC) accounts for about $80 \%$ of differentiated thyroid cancer. ${ }^{(7)}$ Although PTC generally has a relatively indolent course, recurrent disease is not uncommon. It is estimated that $5 \%-20 \%$ of patients develop local/regional recurrences, sometimes after many years have passed. ${ }^{(8)}$ The risk of recurrence is higher in the following instances: older age, male gender, larger tumours, tumours that extend beyond the capsule, certain histological variants, or regional nodal metastases. $^{(8-10)}$

\section{Importance of post-thyroidectomy neck US for surveillance}

Although stimulated thyroglobulin (Tg) testing for recurrent thyroid cancer generally provides effective surveillance against disease recurrence, with a high negative predictive value (NPV) of $99 \%,{ }^{(11)}$ it has a major limitation - interference from $\mathrm{Tg}$ antibodies that cause a falsely low or undetectable serum Tg level can mask the presence of disease. Tg testing is also less useful in patients who have not undergone RAI remnant ablation. Thus, the role of neck US surveillance becomes particularly relevant in these patients and in the context of recurrent disease that is not I-131 avid (which comprises one-quarter of all recurrences).(2)

US is a very sensitive modality for detecting abnormal lymph nodes and masses in the thyroid bed, and may also occasionally demonstrate persistent disease despite a negative stimulated Tg measurement. ${ }^{(12,13)}$ Thus, the American Thyroid Association guidelines have emphasised the role of neck US in the follow-up of patients with PTC. ${ }^{(14)}$ Indeed, the combination

${ }^{1}$ Department of Nuclear Medicine and PET, Singapore General Hospital, Singapore

Correspondence: Dr Sumbul Zaheer, Consultant, Department of Nuclear Medicine and PET, Singapore General Hospital, Outram Road, Singapore 169608. sumbulz@gmail.com 

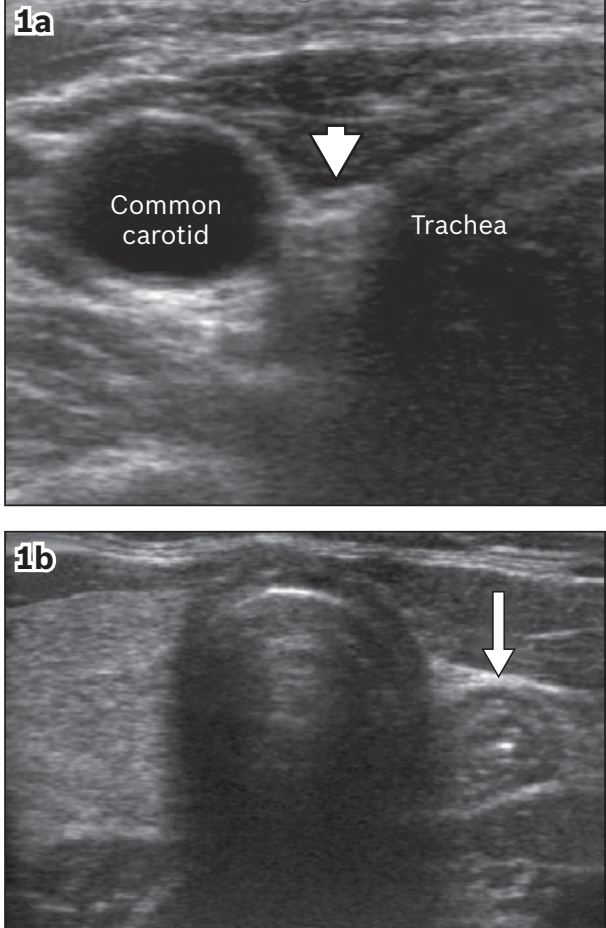

Fig. 1 Normal appearance of the central neck compartment post thyroidectomy. US images show (a) uniform echogenic texture owing to fibrofatty connective tissue in the thyroid bed (arrowhead); and (b) the left thyroid bed occupied by the oesophagus (arrow).

of stimulated Tg and neck US has been found to have a $96.3 \%$ sensitivity in detecting locoregional disease, as well as an NPV of $99.5 \% .^{(15)}$

\section{CENTRAL NECK COMPARTMENT ON POST THYROIDECTOMY US \\ Normal findings and/or benign variants in the central neck}

The central compartment, which contains the thyroid bed and the paratracheal/paraoesophageal nodes, essentially comprises the area between the trachea and carotid arteries. After thyroidectomy, the carotid artery and jugular vein slide medially into the space previously occupied by the thyroid gland. ${ }^{(16)}$ The right carotid artery typically lies immediately adjacent to the trachea (Fig. 1a), whereas the left carotid artery lies along the lateral edge of the oesophagus (Fig. 1b). ${ }^{(16)}$ The normal postoperative thyroid bed should have a uniform echogenic texture owing to fibrofatty connective tissue. ${ }^{(2)}$ Although there is usually no intervening tissue between the trachea and common carotid artery, the finding of uniform echogenic texture owing to fibrofatty connective tissue in the thyroid bed is common (Fig. 1a). The left thyroid bed is often occupied by the oesophagus (Fig. 1b).

The US finding of nodularity in the post-thyroidectomy bed is generally suspicious. However, nodules that contain linear internal echoes parallel to the surrounding tissue plane on longitudinal section (as in Fig. 2b) are most likely due to postoperative granulation tissue. This US appearance has $100 \%$ specificity for benignity. (2) Benign lesions causing
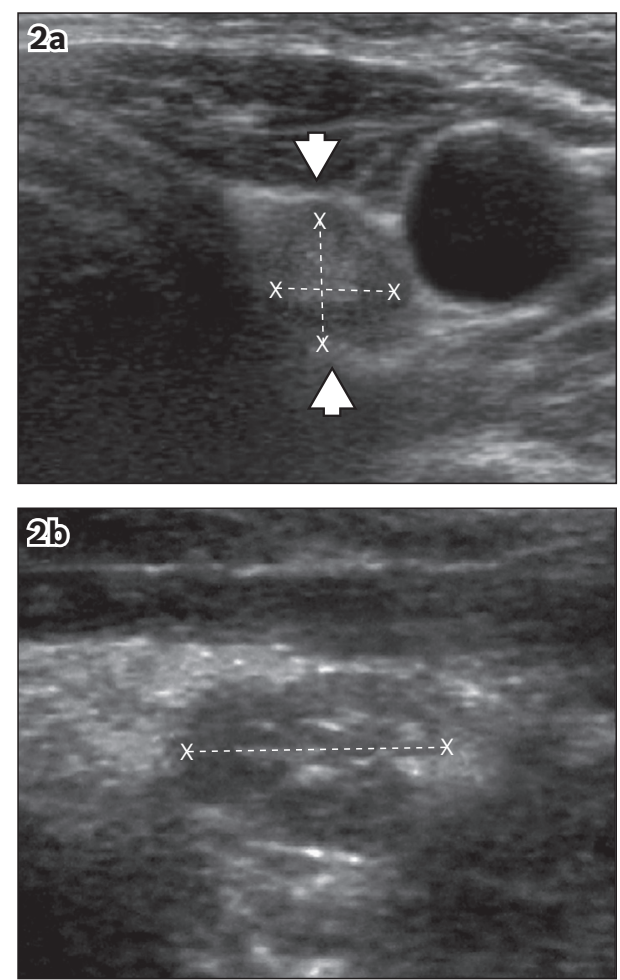

Fig. 2 Benign thyroid bed nodule. US images show (a) a benign thyroid bed nodule (arrowheads) in a patient whose histopathology was unavailable, but the nodule was assumed to be benign, as the patient's thyroglobulin level had been $0.2 \mathrm{UG} / \mathrm{L}$ for at least 6 years; and (b) a nodule that contains linear internal echoes that are parallel to the surrounding tissue plane on longitudinal section.

nodules in the thyroid bed may be due to postoperative fibrosis, suture granulomata, reactive lymph nodes or parathyroid adenoma. ${ }^{(16)}$ Another benign pathology that mimics tumour recurrence in the thyroid bed is remnant thyroid tissue. ${ }^{(2)}$

\section{Features suspicious for malignancy in the central neck}

A recurrence in the thyroid bed or central neck compartment may be due to a true thyroid bed recurrence or recurrent disease in level VI nodes, with the distinction being made by the pathologist only when lymphoid tissue is seen around the cancer cells. ${ }^{(16)}$ Thyroid bed recurrences are typically round and hypoechoic, and situated between the carotid and trachea (Fig. 3a). ${ }^{(16)}$ Most have well-defined margins and some may have microcalcifications or a cystic component (Fig. 4). ${ }^{(16)}$ However, metastases from PTC may appear hyperechoic due to the deposition of Tg (Figs. 3b \& c). Any hypoechoic mass detected in the postoperative thyroid bed is suggestive of recurrence, and a biopsy should be performed, as recurrence is associated with increased mortality. ${ }^{(2)}$ However, if there is no supporting clinical or laboratory finding suggestive of local tumour recurrence, then the abnormality in the thyroid bed may be followed up with US, rather than immediate US-guided fine-needle aspiration cytology. ${ }^{(2)}$ The rate of malignancy is significantly higher in nodules with marginal irregularity (Fig. 4), microcalcification, and a shape that is not parallel to the surrounding tissue plane (Fig. 3c). ${ }^{(2)}$ 

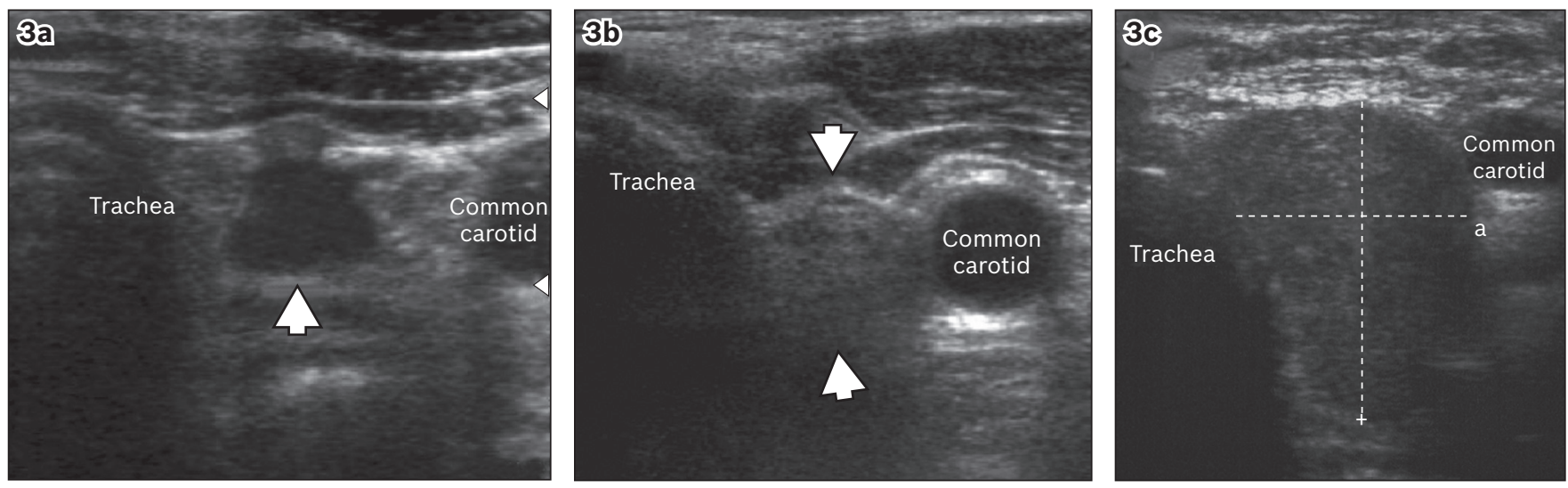

Fig. 3 US images show central neck compartment findings suspicious for malignancy. Subsequent biopsy confirmed recurrence. US images show (a) round, hypoechoic nodules (arrow), typical of recurrences, situated between the carotid artery and trachea; and (b \& c) the hyperechoic appearance of metastases from papillary carcinoma of thyroid (arrowheads) due to the deposition of thyroglobulin. Recurrence shown in (c) was subsequently confirmed to be the insular type of thyroid carcinoma on histopathology.

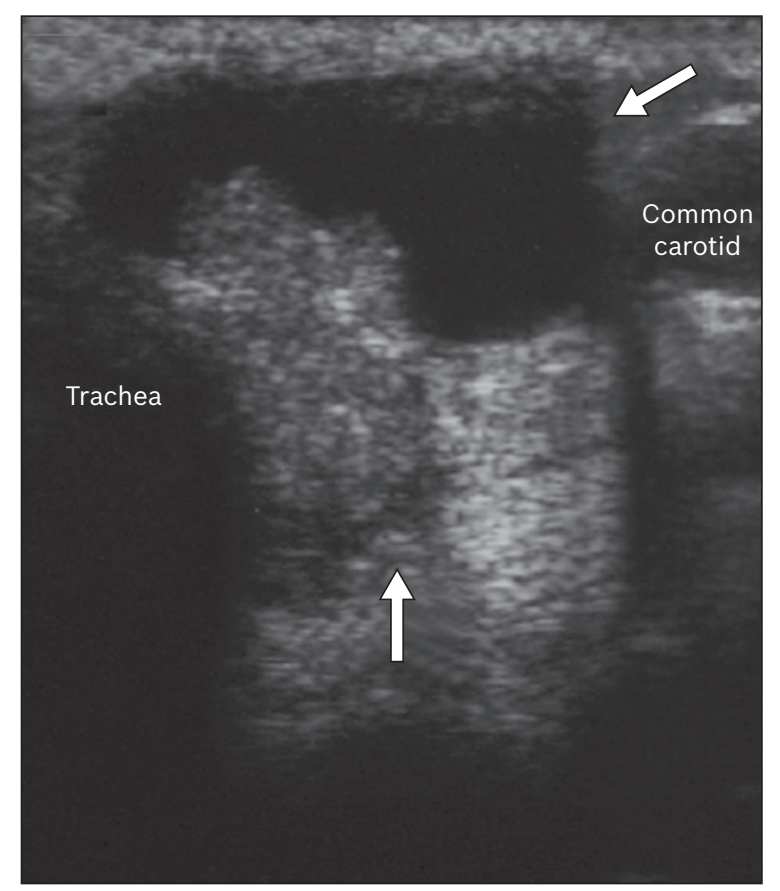

Fig. 4 Histopathology-proven recurrent papillary thyroid carcinoma. US image shows central neck recurrences with heterogeneous internal echoes and cystic components (arrows). Cystic necrosis, as shown here, is more frequently seen in papillary carcinomas.

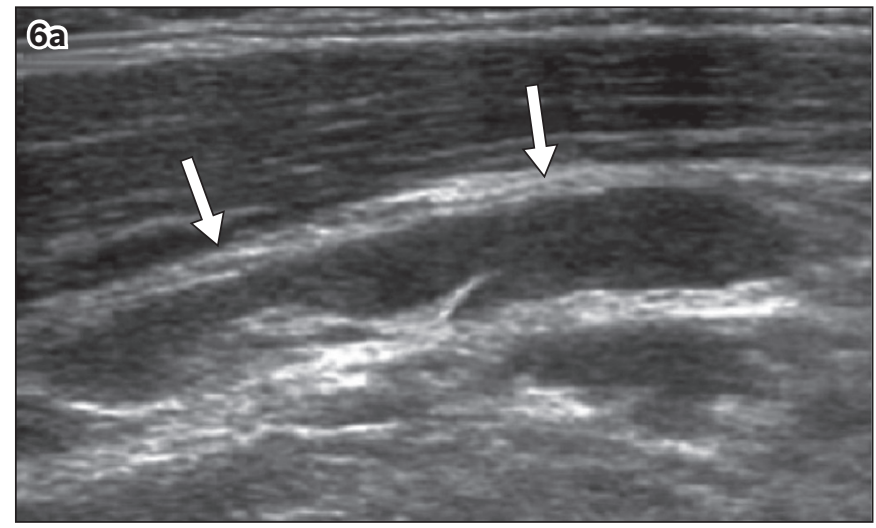

Fig. 6 (a \& b) US images show a $24 \mathrm{~mm} \times 10 \mathrm{~mm} \times 3 \mathrm{~mm}$ benign hyperplastic lymph node (arrows) with its vascular supply entering the fatty hilum.

Fig. 5 Benign lymph nodes. (a) US image shows normal nodes and reactive hyperplastic nodes characterised by an elongated shape and a well-defined echogenic hilum, which is continuous with the adjacent fat (black arrowhead). (b) Power Doppler image shows vascularity entering the centre or hilum of the node and extending toward each tip.

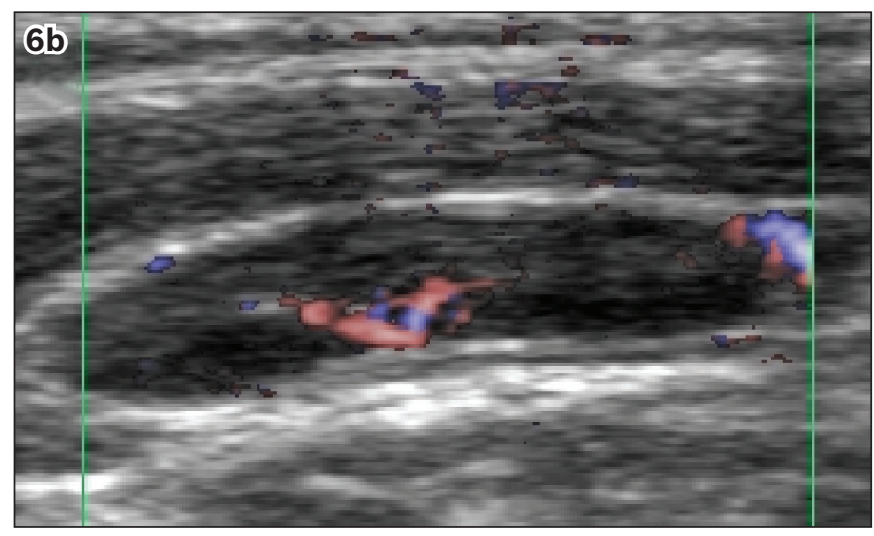




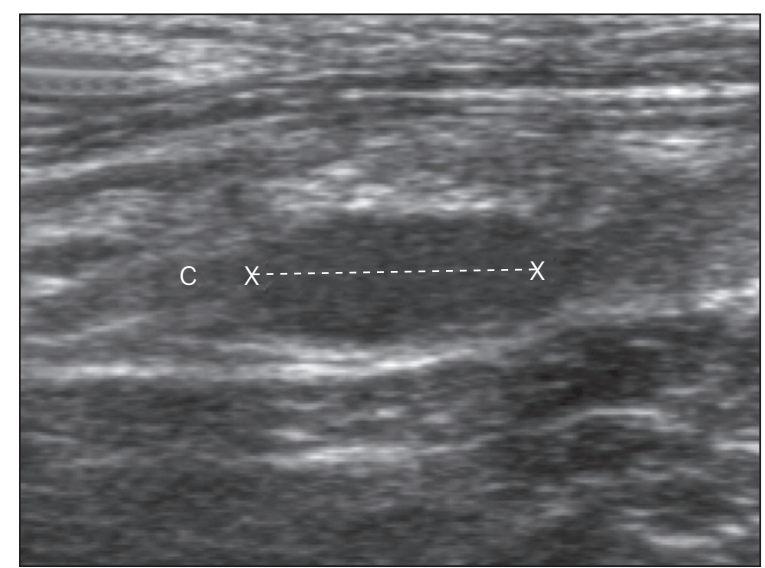

Fig. 7 US image shows a small elongated node measuring less than $5 \mathrm{~mm}$, which can be assumed to be benign. An echogenic fatty hilum may not be identifiable in small nodes, particularly those that are less than $5 \mathrm{~mm}$. These small elongated nodes are commonly encountered in clinical practice and can be assumed to be benign.

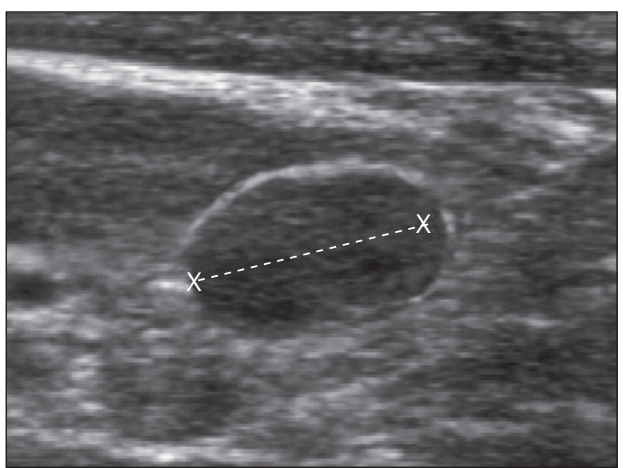

Fig. 8 US image shows a small and somewhat rounded node (in contrast to the elongated shape of a benign node), which may indicate indeterminate or not completely benign-appearing nodes. Also, these nodes may not show the echogenic hilum. When such indeterminate findings are encountered, a definite course of action may not be apparent. Management decisions should incorporate the patient's risk profile and serum thyroglobulin levels. A practical decision-making flow-chart is presented in Fig. 9.

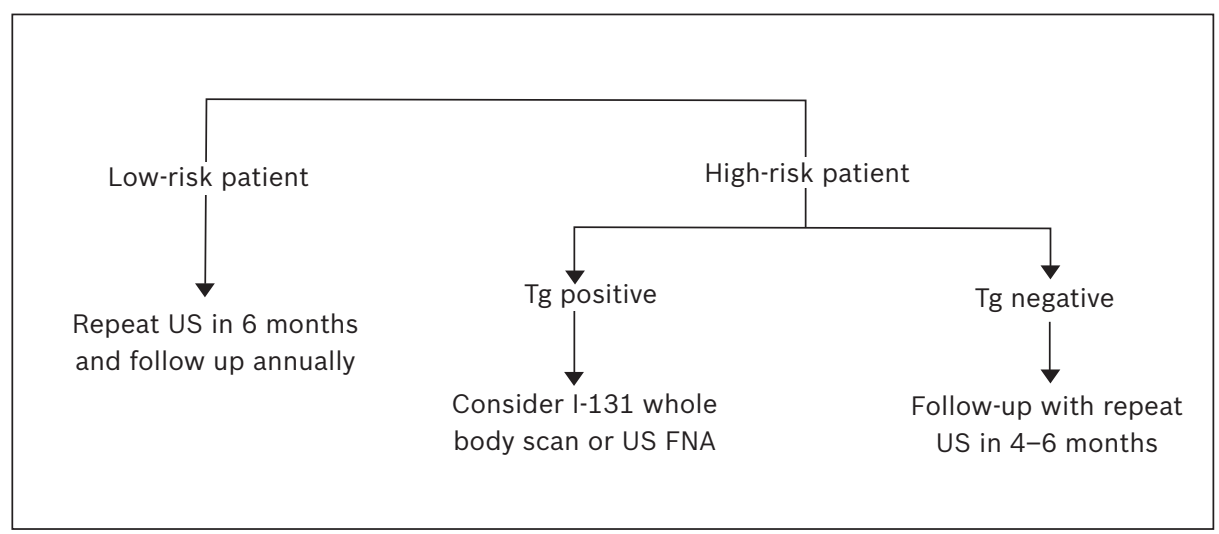

Fig. 9 Flowchart of patient management of indeterminate/not completely benign nodes. ${ }^{(16)}$

FNA: fine-needle aspiration; Tg: thyroglobulin; US: ultrasonography

\section{CERVICAL NODES IN THE NECK ON POST- THYROIDECTOMY US}

\section{Features of benign/reactive nodes}

Lymph nodes with an echogenic centre are typically benign, as are oval or cylindrical nodes. ${ }^{(1)}$ The echogenic centre represents the fatty hilum. Normal nodes and reactive hyperplastic nodes are characterised by an elongated shape and a well-defined echogenic hilum, which is continuous with the adjacent fat (Figs. 5a \& 6a). Power Doppler shows vascularity entering the centre or hilum of the node, and extending toward each tip (Figs. 5b \& 6b). In smaller nodes, an echogenic fatty hilum may not be identifiable, especially in those that measure less than $5 \mathrm{~mm}$. These small elongated nodes are commonly encountered in clinical practice and can be assumed to be benign (Fig. 7).

\section{Features suspicious for malignant nodes, with special reference to differentiated thyroid cancer}

Metastatic nodes are usually hypoechoic (Fig. 8). However, metastatic nodes from PTCs tend to be hyperechoic (Fig. 10a). This is due to the deposition of Tg. ${ }^{(17)}$ Also, the rounder the node, the greater the risk of involvement (Figs. 8 \& 10a). ${ }^{(1)}$ While nodal calcification is generally rare in metastatic nodes, it is common in metastatic nodes from PTCs (Figs. 10a \& b). ${ }^{(17)}$
Lymph nodes showing peripheral vascularity that does not arise from the hilar vessels, or a combination of peripheral and hilar vascularity, are highly suspicious for malignancy (Figs. $11 \& 12 b)$. These peripheral or capsular vessels are recruited in response to pro-angiogenesis factors released by tumour cells. ${ }^{(17)}$ Thus, signs of malignant adenopathy include the presence of internal calcification, a combination of peripheral and hilar vascularity (an almost certain sign of recurrent papillary cancer) (Figs. $11 \& 12 \mathrm{~b}$ ), cystic or partially cystic nodes (Fig. 12a) and echogenic nodes. ${ }^{(1)}$

Recognition of advanced nodal disease with local infiltration of adjacent structures cannot be missed, as shown in Fig. 13. In a patient with PTC, the longitudinal US image shows an echogenic mass within the lumen of the right internal jugular vein (Fig. 13a), while the correlative computed tomography image confirms the infiltration of the right internal jugular vein by adjacent metastatic lymph node (Fig. 13b). Tumour recurrence in the right thyroid bed is also noted.

Among the features described, Leboulleux et al reported the specificities for malignancy to be $100 \%$ for cystic appearance and punctate calcifications, although the corresponding sensitivity values were low $(46 \%$ and $11 \%$, respectively). ${ }^{(18)}$ In the same study, the US feature that gave the 

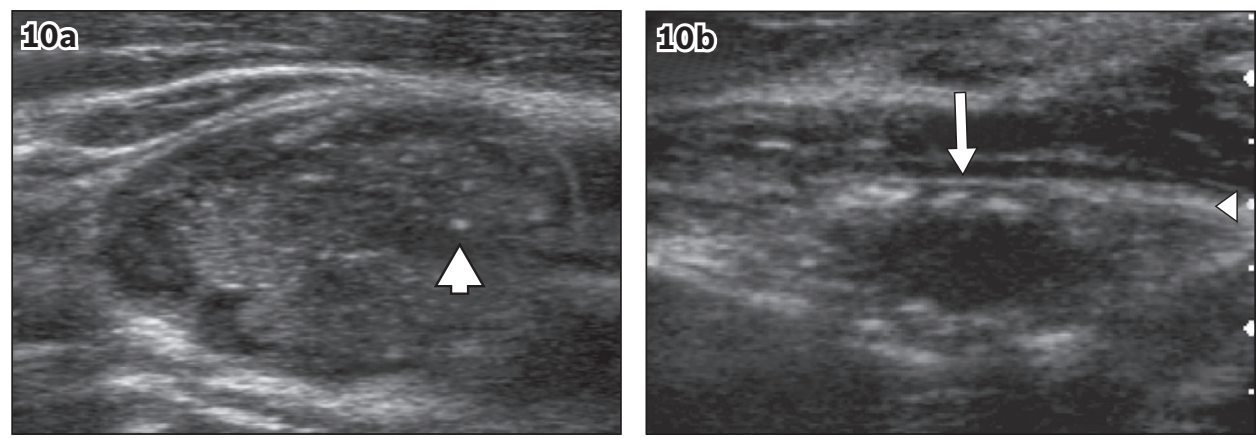

Fig. 10 US images show (a) nodal calcification in metastatic nodes from papillary thyroid cancer, which is usually fine or punctate (arrowhead), representing calcified psammoma bodies; and (b) metastatic lymph node with ill-defined margin, containing peripheral calcifications (arrow). Nodes suspicious for malignancy may show the following features: presence of calcifications, cystic or necrotic change, rounded or irregular shape, inhomogeneous internal echopattern, abnormal vascularity, and loss of echogenic hilum.

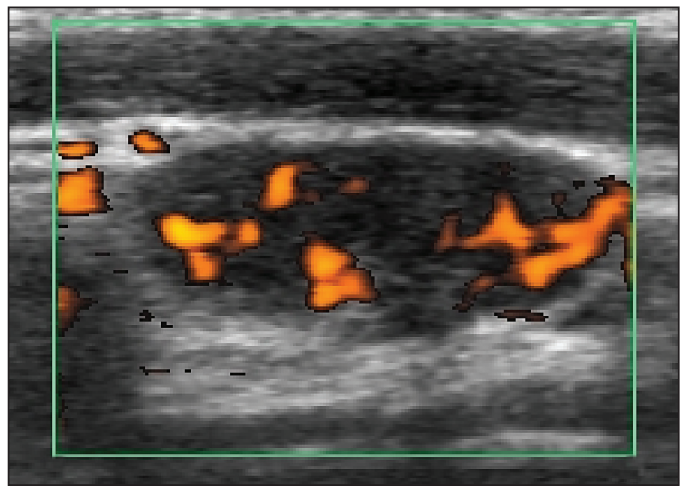

Fig. 11 US image shows a lymph node with a combination of peripheral and hilar vascularity, which is highly suspicious for malignancy. Nodes showing peripheral vascularity that does not arise from the hilar vessels or a combination of peripheral and hilar vascularity, are highly suspicious for malignancy. These peripheral or capsular vessels are recruited in response to pro-angiogenesis factors released by tumour cells. ${ }^{(17)}$
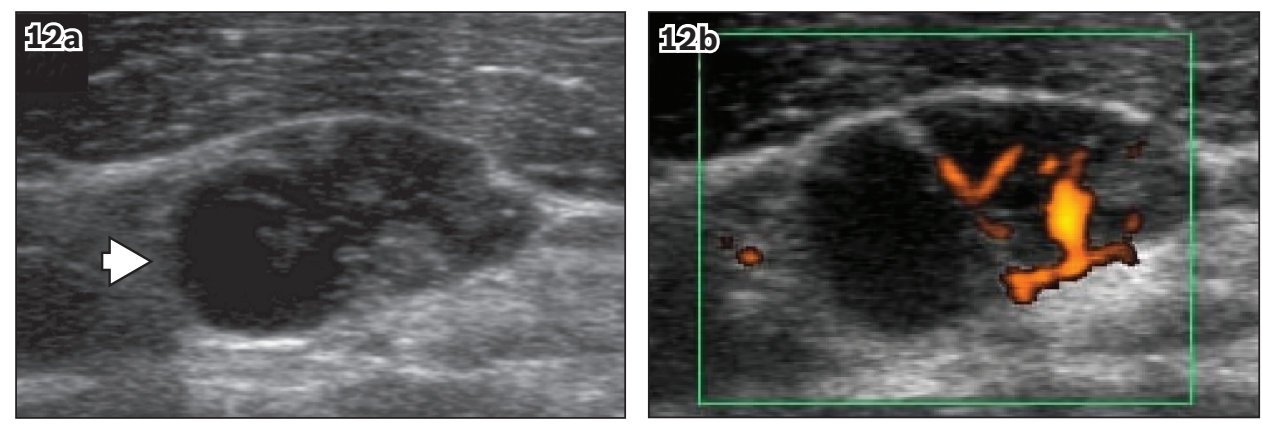

Fig. 12 US images show (a) a malignant lymph node containing a cystic area (arrowhead); and (b) intranodal vascularity being displaced in the presence of cystic necrosis.
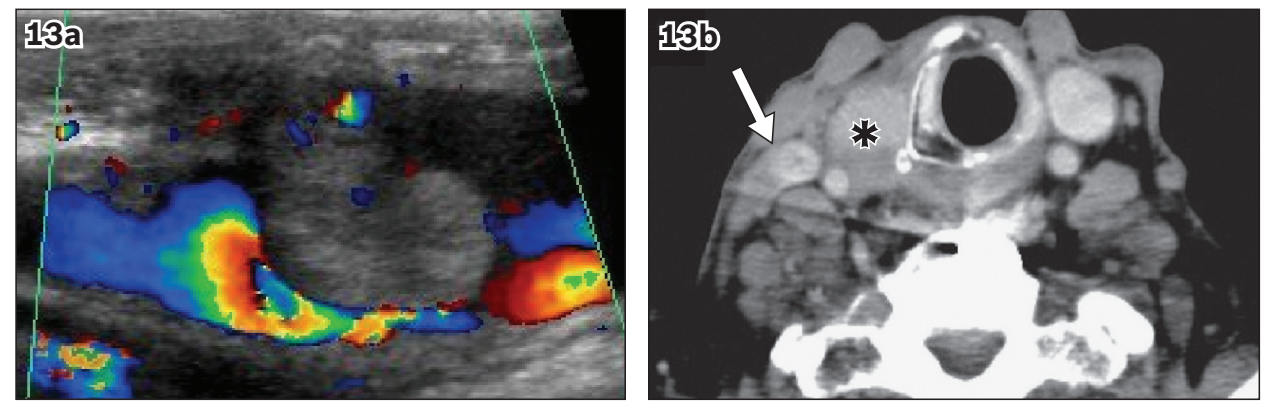

Fig. 13 Advanced nodal disease with local infiltration of adjacent structures is infrequently encountered. (a) Longitudinal US image shows an echogenic mass within the lumen of the right internal jugular vein in a patient with papillary thyroid cancer. (b) Correlative CT image confirms the infiltration of the right internal jugular vein by adjacent metastatic lymph node (arrow). Tumour recurrence in the right thyroid bed is apparent (asterisk). 
best sensitivity-specificity compromise $(86 \%$ and $82 \%$, respectively) was the pattern of intranodal vascularisation.

\section{CONCLUSION}

Neck US is a simple, noninvasive and highly sensitive tool for monitoring disease recurrence in post-thyroidectomy patients with differentiated thyroid cancer. Knowledge of the differentiating features of benign and malignant lesions in these patients may significantly increase the efficacy of this investigation. Along with serum Tg levels and I-131 whole body imaging, neck US should be the cornerstone in the follow-up of post-thyroidectomy patients with thyroid cancer.

\section{REFERENCES}

1. Jung AS, Grant EG. Ultrasound interventions in the neck with emphasis on postthyroidectomy papillary carcinoma. Ultrasound Clin 2009; 4:1-16.

2. Lee $\mathrm{JH}$, Lee $\mathrm{HK}$, Lee $\mathrm{DH}$, et al. Ultrasonographic findings of a newly detected nodule on the thyroid bed in postoperative patients for thyroid carcinoma: correlation with the results of ultrasonography-guided fineneedle aspiration biopsy. Clin Imaging 2007; 31:109-13.

3. Cooper DS, Doherty GM, Haugen BR, et al. Revised American Thyroid Association management guidelines for patients with thyroid nodules and differentiated thyroid cancer. Thyroid 2009; 19:1167-214.

4. British Thyroid Association. Guidelines for the management of thyroid cancer. 2nd ed. London: British Thyroid Association and Royal College of Physicians, 2007.

5. Singapore Registry of Diseases Office. Singapore Cancer Registry Interim Annual Registry Report: Trends in Cancer Incidence in Singapore 20052009 [online]. Available at: https://www.nrdo.gov.sg/uploadedFiles/ NRDO/Cancer_Trends_Report\%20_05-09.pdf. Accessed April 4, 2014.

6. Cheah WK. Thyroid cancer: diagnosis and management. Singapore Med J 2007; 48:107-12.
7. Hundahl SA, Cady B, Cunningham MP, et al. Initial results from a prospective cohort study of 5583 cases of thyroid carcinoma treated in the united states during 1996. US and German Thyroid Cancer Study Group. An American College of Surgeons Commission on Cancer Patient Care Evaluation study. Cancer 2000; 89:202-17.

8. Schlumberger MJ. Papillary and follicular thyroid carcinoma. N Engl J Med 1998; 338:297-306.

9. Bardet S1, Malville E, Rame JP, et al. Macroscopic lymph-node involvement and neck dissection predict lymph-node recurrence in papillary thyroid carcinoma. Eur J Endocrinol 2008; 158:551-60.

10. Ito Y1, Higashiyama T, Takamura Y, et al. Risk factors for recurrence to the lymph node in papillary thyroid carcinoma patients without preoperatively detectable lateral node metastasis: validity of prophylactic modified radical neck dissection. World J Surg 2007; 31:2085-91.

11. Schlumberger M, Berg G, Cohen O, et al. Follow-up of low-risk patients with differentiated thyroid carcinoma: a European perspective. Eur J Endocrinol 2004; 150:105-12.

12. Frasoldati A, Pesenti M, Gallo M, et al. Diagnosis of neck recurrences in patients with differentiated thyroid carcinoma. Cancer 2003; 97:90-6.

13. Torlontano M, Attard M, Crocetti U, et al. Follow-up of low risk patients with papillary thyroid cancer: role of neck ultrasonography in detecting lymph node metastases. J Clin Endocrinol Metab 2004; 89:3402-7.

14. ATA Guidelines Taskforce on Thyroid Nodules and Differentiated Thyroid Cancer, Cooper DS, Doherty GM, Haugen BR, et al. Revised American Thyroid Association management guidelines for patients with thyroid nodules and differentiated thyroid cancer. Thyroid 2009; 19:1167-214.

15. Pacini F, Molinaro E, Castagna ME, et al. Recombinant human thyrotropinstimulated serum thyroglobulin combined with neck ultrasonography has the highest sensitivity in monitoring differentiated thyroid carcinoma. J Clin Endocrinol Metab 2003; 88:3668-73.

16. Frates MC. Ultrasound in recurrent thyroid disease. Otolaryngol Clin North Am 2008; 41:1107-16.

17. Ahuja A and Ying M. Sonography of neck lymph nodes. Part II: abnormal lymph nodes. Clin Radiol 2003; 58:359-66.

18. Leboulleux S, Girard E, Rose M, et al. Ultrasound criteria of malignancy for cervical lymph nodes in patients followed up for differentiated thyroid cancer. J Clin Endocrinol Metab 2007; 92:3590-4. 


\section{SINGAPORE MEDICAL COUNCIL CATEGORY 3B CME PROGRAMME}

\section{(Code SMJ 201404A)}

Question 1. The most commonly encountered form of thyroid cancer is:

(a) Papillary thyroid cancer.

(b) Medullary thyroid cancer.

(c) Follicular thyroid cancer.

(d) Anaplastic thyroid cancer.

Question 2. In differentiated thyroid cancer, the following is/are associated with an increased risk of recurrence:
(a) Male gender.
(b) Younger age at diagnosis.
(c) Presence of nodal metastases at diagnosis.
(d) Invasion of thyroid capsule by tumour.

Question 3. Following total thyroidectomy, locoregional recurrence of papillary thyroid cancer is suspected in patients with:
(a) Elevated serum thyroid-stimulating hormone.
(b) Elevated serum thyroglobulin.
(c) Elevated serum calcitonin.
(d) Presence of a solid cystic mass in the thyroid bed on ultrasonography (US).

Question 4. Regarding US for surveillance of thyroid cancer recurrence:
(a) US has a high sensitivity for detecting locoregional recurrence.
(b) US is not useful if serum thyroglobulin is normal.
(c) Benign lymph nodes typically appear elongated.
(d) All lymph nodes $>1 \mathrm{~cm}$ require fine-needle aspiration evaluation.

Question 5. Features of malignant lymph nodes on US include:
(a) Presence of calcifications.
(b) Presence of cystic areas.
(c) Rounded shape.
(d) Displaced intranodal vascular flow.

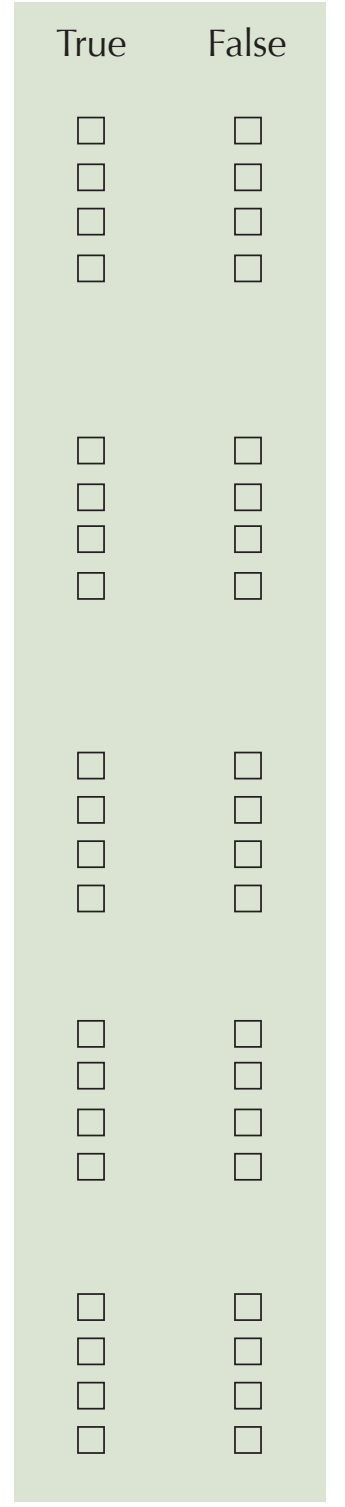

\footnotetext{
Doctor's particulars:

Name in full

MCR number

Specialty:

Email address

(1) Log on at the SMJ website: http://www.sma.org.sg/publications/smjcurrentissue.aspx and select the appropriate set of questions. (2) Provide your name, email address and MCR number. (3) Select your answers and click "Submit".

RESULTS:

(1) Answers will be published in the SMJ June 2014 issue. (2) The MCR numbers of successful candidates will be posted online at the SMJ website by 23 May 2014. (3) Passing mark is $60 \%$. No mark will be deducted for incorrect answers. (4) The SMJ editorial office will submit the list of successful candidates to the Singapore Medical Council. (5) One CME point is awarded for successful candidates.

Deadline for submission: (April 2014 SMJ 3B CME programme): 12 noon, 16 May 2014.
} 